\title{
RECEIPT AND DISPATCH OF AN AIRCRAFT: A FUNCTIONAL RISK ANALYSIS
}

\author{
Riccardo Patriarca ${ }^{I}$ \\ Arie Adriaensen ${ }^{1}$ \\ Mark Peters ${ }^{2}$ \\ Joel Putnam ${ }^{2}$ \\ Francesco Costantino $^{1}$ \\ Guilio Di Gravio ${ }^{1}$ \\ 1) Department of Mechanical and Aerospace Eng. Sapienza University of Rome, Italy \\ 2) Safety Quality \& Compliance Qantas Engineering, Qantas Airways Limited, Australia
}

\begin{abstract}
Receipt and dispatch operations in aviation require the contribution of a variety of tightly interconnected agents (dispatchers, pilots, ground-handlers, air traffic controller, etc.). These agents have to deal with processes that are frequently affected by variable working conditions and limited resources. In this context, human actions acquire a crucial role to cope with situations underspecified by procedures, and to manage unanticipated circumstances.

This paper proposes the application of the Functional Resonance Analysis Method (FRAM) for modelling receipt and dispatch process for Boeing-737 in a major airport (about 70 movements per day). The proposed FRAM has been enriched by the adoption of a multi-level representation aimed at deconstructing the complexity of work at different abstraction levels. The analysis focuses on agents and macro-meso-micro functions (i.e. functions aggregated into a single function at a higher abstraction level), combining qualitative and semi-quantitative analyses. This paper aims to detail the main project phases for the development of a FRAM model to be used as a basis for systemic risk analyses in large socio-technical systems.
\end{abstract}

Keywords: system safety, socio-technical system, airport safety, aviation safety, airline safety

\section{CONTEXT}

Over years, airport operations became increasingly more complex in terms of interactions, number of components, level of automation, and congestion. For such complex processes, the contribution of human operators remains necessary, mainly for those tasks where adaptation is a crucial factor to guarantee the feasibility of the operation.

Adopting a socio-technical point of view, Resilience Engineering (RE) aims to develop complexity-oriented analyses of processes, (e.g.) focusing on the differences between work-asdone and work-as-imagined (at least among other archetypes of work) [1].

(C) 2019 Patriarca, Adriaensen, Peters, Putnam, Constantino \& Di Gravio. This is an Open Access article distributed under the terms of the Creative Commons Attribution-NonCommercial 4.0 International License

(http://creativecommons.org/licenses/by-nc/4.0), permitting all non-commercial use, distribution, and reproduction in any medium, provided the original work is properly cited.

ISBN: 978-91-88898-41-8

DOI: https://doi.org/10.15626/rea8.17 
RE-driven analyses are intended with no preconceptions for the characterization of human beings as flawed components of the system, but rather assuming their adaptive capacities as crucial for the process' success.

Over recent years, Qantas Engineering (QE) has been pursuing a strategic objective of becoming a High Reliability Organisation (HRO). HROs are organisations that: (i) conduct relatively error-free activities over a long period of time; (ii) deliver high quality and reliable operations consistently; and (iii) operate in industries where failure may have potentially catastrophic consequences [2]. Extending the HRO notion of reliability, i.e. reliability of processes against failures, QE is also embracing a path to resilience, i.e. robustness against variability. In particular, guiding principles for achieving these targets include:

- Manage the system not the parts

- Manage system variability

- Foster a strong culture of reliability

- Learn and adapt as an organisation

Following this vision, the project summarized in this manuscript aims at developing an experimental analysis for an airline operation, through the adoption of a RE tool, i.e. the Functional Resonance Analysis Method (FRAM).

The process selected for the research was aircraft Receipt and Dispatch (see section 3 for a detailed process description). This process was selected due to a recent increase in related incidents, and perceived functional complexity across multiple procedures, locations and fleet types. Consequently, it was considered a functional area with opportunity for improvement.

The remainder of the paper is organized as follows: Section 2 details the features of the case study, Section 3 briefly presents the method adopted, which is discuss in detail in Section 4, with the ultimate aim to support its applicability in a variety of industries.

\section{CASE STUDY}

Aircraft Receipt \& Dispatch is the function of an aircraft arriving and departing a parking bay at the Airport Terminal, including marshalling (if required), aircraft arrival on the parking bay and shutdown; and then pre-flight (walk-around) inspection, start-up and pushback prior to the aircraft taxying to the runway under its own power.

During the dispatch (pushback) routine, two people are currently required to be available due to the use of conventional pushback tugs utilising towbars. One person remains in control of the tug, while the other is utilised to connect / disconnect the towbar from the tug / aircraft.

Separate to the Receipt and Dispatch process is that of Aircraft Towing which relates to movement of aircraft between locations on the airport - e.g. from an engineering facility to the airport terminal, and which was outside the scope of this study. Also out of scope was other elements of the process related to the aircraft turnaround including passenger boarding / deboarding, loading of baggage, freight and catering, and completion of paperwork such as flight plan, loadsheet, and passenger manifest, etc.

The main components of the process under analysis are thus:

- Arrival operation

- Preparation for dispatch

- Pushback operation

Other supporting components include:

- Logistical and organisational support

- Contingency management

Figure 1 summarize the relations between main components of the process. 


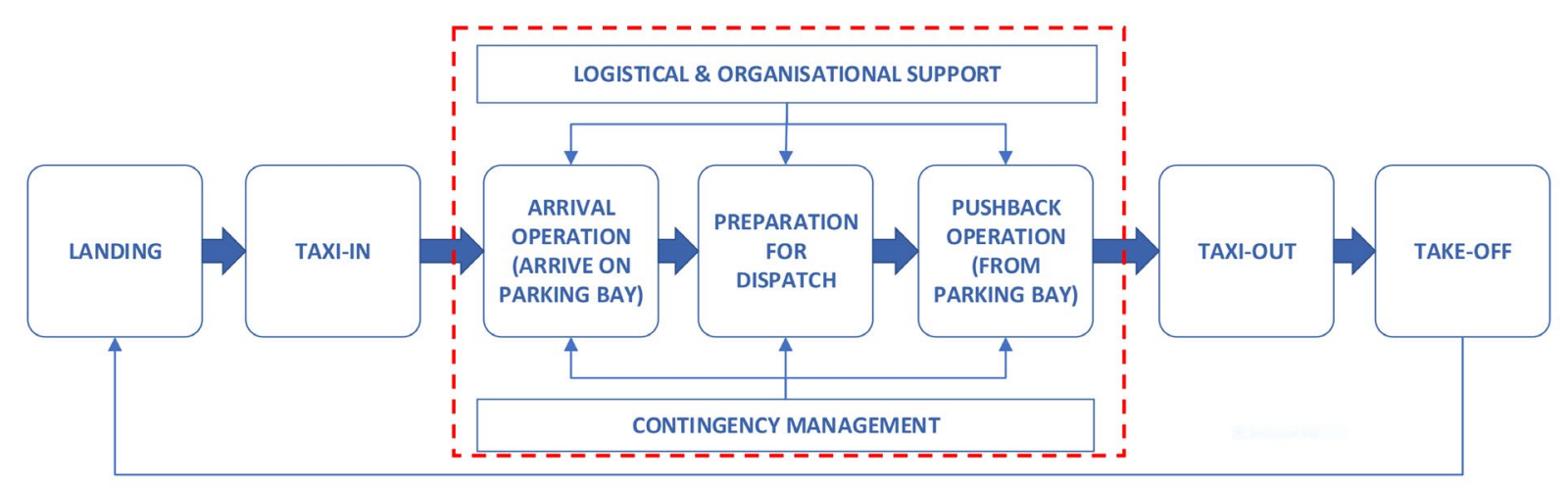

Figure 1. Main phase of the receipt and dispatch process.

The main actors for the process are as follows:

- Person Departing Aircraft (PDA). This role communicates with the flight deck and the Pushback Driver during the departure of the aircraft from the parking bay, and is physically near the aircraft during that process, usually walking on the tarmac next to the aircraft while it is pushed-back. They alert the Flight Crew of any emergency/ nonnormal situations, ensuring clear and accurate 'two way' communication of information from ground crew to the Pilot-in-Command to ensure that the Pilot-in-Command can determine the safest course of action for passengers and Air Crew during a non-normal situation.

- Person Arriving Aircraft (PAA). This role 'greets' the aircraft and communicates with the flight deck on arrival of the aircraft at the parking bay. The role places chocks in front and behind the aircraft tyres, advises other ground crew when it is safe to approach the aircraft, plugs-in ground power and air conditioning etc. This person may or may not be the same person as that conducting the PDA role.

- Pilot. Pilot of the aircraft located on the flight deck.

- Pushback Driver. Driver of the vehicle that pushes the aircraft away from the Terminal. Physically located in the vehicle. Takes direction from the PDA.

- Air Traffic Control (ATC). ATC provides clearance to the aircraft to commence pushback and to taxi on the taxiway.

\section{METHOD}

The method applied in this research is the Functional Resonance Analysis Method (FRAM) [3], aimed at providing a methodological support for modelling the varieties of the work domain under investigation. The FRAM has been used for modelling the work-as-imagined, in line with documents and then the work-as-done, as emerged from interviews, and observational studies. These labels remain valid under the assumption that, through multiple data sources, the bias inferred in the model by the role of the observer can be considered of minor entity for the development of a credible representative model.

The research relied on document analysis to create a representative context for the model to be developed (e.g., procedures, handbooks, relevant incident reports, etc.). Such analysis constituted the basis for the development of a work-as-imagined model of the receipt and dispatch process.

Then the results of the document analysis have been validated through semi-structured individual interviews with people involved in the process at different level. A series of observational studies were performed to validate the results of the interviews and gather additional evidence on the process. 
In methodological terms, the developed FRAM followed a multi-layer perspective, in line with the Abstraction/Agency framework in order to generate clearer and more representative model(s). The same model, once gathered data from interviews and observations allowed the definition of a semi-quantitative model used to run a Monte Carlo simulation of the most significant functional parameters.

\section{PROJECT PHASES}

The project has been articulated in 7 phases, as depicted in Figure 2, further specifying the applied methodological approach.

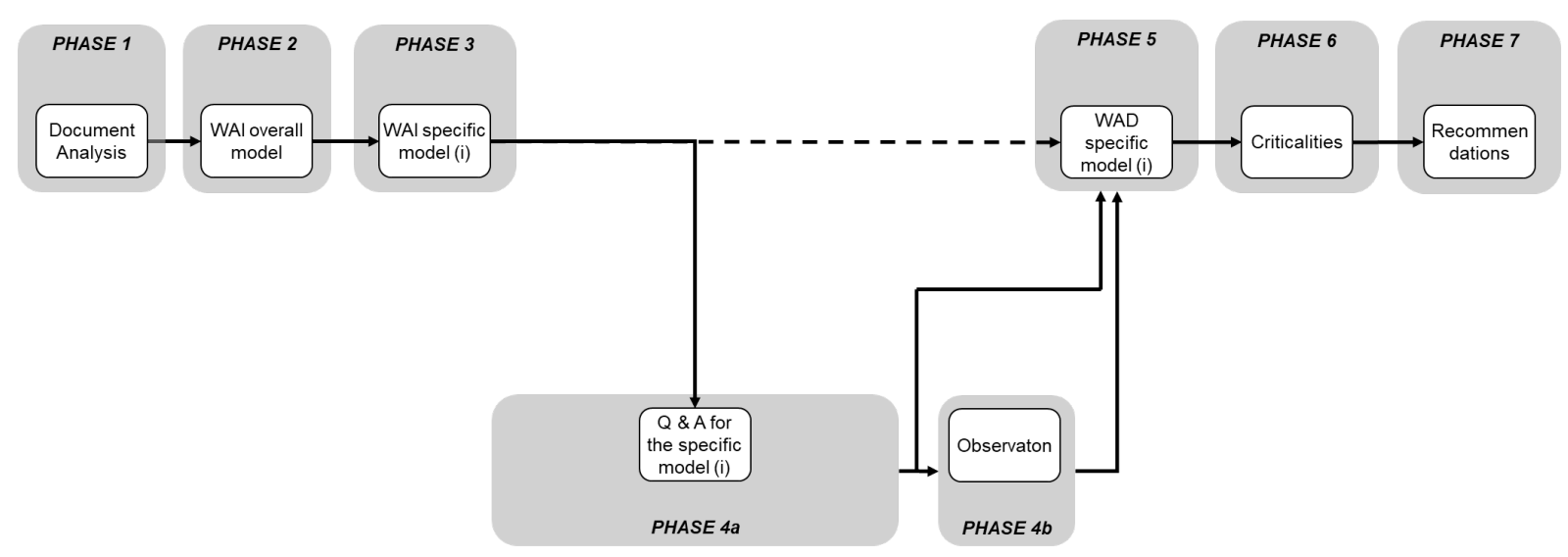

Phase 1

Figure 2. Main phase of the process.

As already introduced in Section 3, an initial document analysis was performed, based on manuals and procedures from different flight and ground crew actors, jointly involved in the distributed socio-technical processes at hand. In addition, incident report data was examined to gain un in-depth understanding of critical occurrences, either for frequency or because of their potential to create substantial harm. From this, data-driven criticalities were retained for further use in Phase 3.

Phase 2

A high-level Hierarchical Task Analysis (HTA) model was developed to serve as the basis for an overall work-as-imagined FRAM model that contained all of the procedural FRAM functions for Aircraft Receipt \& Dispatch, as detailed in the company procedures. The HTA served as a raw sum-up and decomposition of work activities to be introduced in a first very reductionist FRAM model, also called a raw FRAM model. HTA as a preliminary step to facilitate further functional FRAM analysis has previously been used in literature [3]-[5].

\section{Phase 3}

The raw FRAM model became the basis to identify model-driven criticalities, when looking at procedural vulnerabilities. These were compared with the data-driven criticalities, derived from incident report data, retained in Phase 1. A legend with criticality labels was designed and assigned to the identified criticalities, where necessary. Assigned labels were:

- 'ambiguous' or 'inconsistent' processes from a work-as-imagined perspective

- 'incident report link', defining a match between model-driven and data-driven (i.e. validated by an occurrence) criticality

- 'emergent behaviour', which is local performance variability from incident report data that could not be explained by existing procedural documentation, and finally 
- a 'clarifications needed' label, requiring further informant clarification The most critical scenarios were selected for further work-as-done examination, introduced in myFRAM [6] and converted with the FRAM Model Visualiser (FMV) [7].

\section{Phase 4}

Phase 4a) Once the raw FRAM model and several critical sub-models were derived, semistructured interviews were prepared in order to further investigate the criticalities discovered from Phase 3, although this time from a work-as-done perspective. Interviews were prepared with dispatcher, pushback driver and pilot informants. The process used semi-structured interviews to determine the final list of functions and couplings, specifically applicable in operational contexts, a strategy previously used in other FRAM analyses [8]-[10].

Phase $4 \mathrm{~b}$ ) Simultaneously, the phase served as a basis to resolve remaining criticalities from Phase 3 and Phase 4 a by subsequent observational investigation, previously used in the FRAM literature ([4], p.14). The research project used open-ended naturalistic observations, often labelled as pure observations for their ability to generate the opportunity to observe work without the blinders of hypotheses and other preconceptions [11], also used in FRAM analyses [12], [13]. Observations included timing, sequence, and process in receipt and dispatch of aircraft.

\section{Phase 5}

An equal amount of work-as-done FRAM models have been developed to mirror the work-asimagined FRAM models from Phase 3. Functions and couplings were deleted, edited and added with the data obtained from interviews and observational studies from Phase $4 \mathrm{a}$ and Phase $4 \mathrm{~b}$.

\section{Phase 6}

The criticalities in the work-as-done FRAM models were identified and labelled with a dedicated legend, similarly to what included in Phase 3. Labels included (i) 'variable functions', meaning they showed high variability among different agents; (ii) 'additional WAD functions' that showed a function that emerged from informant or observational data that were richer than the procedural descriptions; (iii) 'absent in WAD', a label for functions described by the procedures but not acknowledged by observational data or validated by informants. The models enriched with the labels provided a means of communication with the airline's safety management for internal validation.

Additionally, a Monte Carlo simulation model was applied to the specific scenario of covering management of towbar failure during pushback. The approach aims at associating a semiquantitative criticality index to each coupling, based on the function internal variability, the upstream/downstream connection, and the effects of relevant operational conditions [14].

The focus on towbar failure during pushback as a simulation scenario, was due to the encountered difficulty when studying the procedural variants from company documents. During aircraft pushback, there is a difference in management of a towbar failure that is completely separated or one that is still attached. The type of failure determines indeed both the physical forces and chronology of actions between flight crew and push back driver, coordinated and mediated by the dispatcher. This led the researchers to require clarifications from informants in Phase 4(b). It was confirmed as a potential critical situation by several informants from both ground and flight crews, and for which there was no uniform knowledge among different informants. Published procedures sometimes differed from local practice. The execution of work-as-done functions sometimes showed a richer set of operational parameters than work-as-imagined. Individual professional knowledge from informants, both from ground and flight crew, offered non-published solutions to simplify procedures for a set of fluctuating operational conditions, otherwise requiring many procedural variants. Therefore, the output of 
this phase supported the design of a Monte Carlo simulation model developed to encompass the work-as-done reality.

\section{Phase 7}

The final step consisted of proposing ways to manage the possible combinations of uncontrolled performance variability and subsequent potential for functional resonance. Recommendations emerged from: gap analysis between work-as-imagined and work-as-done; (ii) previously unknown performance variability derived from semi-structured interviews; (iii) research observations confirmed by detailed analysis of the functional model(s).

\section{CONCLUSION}

The research relied on a systematic assessment of risks using the FRAM. The proposed methodological framework helped identifying criticalities, filling open gaps in work practices and supporting proactively risk management in receipt and dispatch operations. The success of the project was highly dependent on the resources invested by QE, i.e. availability of key persons, possibility to have direct contacts between researchers and sharp-end operators, remote access to key documents and relevant company database. It is worthy notice how the interviews were positively perceived by the operators, who were extremely willing to participate in the business process through their work experience. They contributed significantly to the outcomes of the research. For QE, while the project aims were achieved, there were some key learnings as an organisation:

- Implementation of a tool such as FRAM needs to be supported by adoption of an underlying strategy to look at safety as an outcome of how work will be done in the future. This is because the process (whilst yielding results), is time-intensive and requires focus, and unless existing safety management practices are 'swapped-out' for methods such as FRAM, then essentially all that is happening is an increase in workload for safety practitioners;

- A tool needs to be developed to enable prioritisation of processes for FRAM analysis this is being developed within the business at present.

This project has provided the basis for organisational learning, and despite the comments above, QE objective of becoming a HRO by proactively managing risk is still actively being pursued.

\section{ACKNOWLEDGEMENTS}

The authors want to thank all the operators involved in the interviews and in the observational process: their desire to collaborate, and the information they provided during the data gathering process were crucial for the success of the project, and for the systemic analysis of risks in operations.

\section{REFERENCES}

[1] S. Shorrock, "The varieties of human work," Humanistic Systems blog, 2016. [Online]. Available: https://humanisticsystems.com/2016/12/05/the-varieties-of-human-work/. [Accessed: 25-Apr-2017].

[2] J. Pariès, L. Macchi, C. Valot, and S. Deharvengt, "Comparing HROs and RE in the light of safety management systems," Saf. Sci., no. February, pp. 0-1, 2018.

[3] E. Hollnagel, FRAM: The Functional Resonance Analysis Method - Modelling Complex Socio-technical Systems. Ashgate, 2012.

[4] R. Patriarca and J. Bergström, "Modelling complexity in everyday operations: functional resonance in maritime mooring at quay," Cogn. Technol. Work, vol. 19, no. 4, pp. 711-729, 2017. https://doi.org/10.1007/s10111-017-0426-2 
[5] L. De Vries, "Work as Done? Understanding the Practice of Sociotechnical Work in the Maritime Domain,” J. Cogn. Eng. Decis. Mak., vol. 11, no. 3, pp. 270-295, 2017.

[6] R. Patriarca, G. Di Gravio, and F. Costantino, "MyFRAM: An open tool support for the functional resonance analysis method," in 2017 2nd International Conference on System Reliability and Safety, ICSRS 2017, 2018, vol. 2018-Janua, pp. 439-443.

[7] R. Hill and E. Hollnagel, "Instructions for use of the FRAM Model Visuliser (FMV)," 2016.

[8] L. de Vries and L. O. Bligård, "Visualising safety: The potential for using sociotechnical systems models in prospective safety assessment and design," Saf. Sci., vol. 111, no. October 2017, pp. 80-93, 2019.

[9] G. Praetorius, A. Graziano, J.-U. Schröder-Hinrichs, and M. Baldauf, Fram in FSAIntroducing a function-based approach to the formal safety assessment framework, vol. 484. 2016. https://doi.org/10.1007/978-3-319-41682-3_34

[10] G. Praetorius, E. Hollnagel, and J. Dahlman, "Modelling Vessel Traffic Service to understand resilience in everyday operations," Reliab. Eng. Syst. Saf., vol. 141, pp. 10 21, Sep. 2015. https://doi.org/10.1016/j.ress.2015.03.020

[11] M. Q. Patton, Qualitative research and evaluation methods, 3rd editio. Thousand Oaks, CA: Sage Publications, 2002.

[12] R. Patriarca, "Developing risk and safety management methods for complex sociotechnical systems - from newtonian reasoning to resilience engineering," Sapienza University of Rome, 2017.

[13] G. Praetorius, E. Hollnagel, and J. Dahlman, "Modelling Vessel Traffic Service to understand resilience in everyday operations," Reliab. Eng. Syst. Saf., vol. 141, pp. 1021, Sep. 2015. https://doi.org/10.1016/j.ress.2015.03.020

[14] R. Patriarca, G. Di Gravio, and F. Costantino, "A Monte Carlo evolution of the Functional Resonance Analysis Method (FRAM) to assess performance variability in complex systems," Saf. Sci., vol. 91, 2017. https://doi.org/10.1016/j.ssci.2016.07.016 